\title{
Elevated Expression of the RNA-Binding Protein IGF2BP1 Enhances the Mrna Stability and Translation Efficiency of INHBA to Promote the Invasion and Migration of Esophageal Squamous Cancer Cells
}

\section{Juan-Juan Wang}

Chinese Academy of Medical Sciences \& Peking Union Medical College

\section{Ding-Xiong Chen}

Chinese Academy of Medical Sciences \& Peking Union Medical College

\section{Yu Zhang}

Chinese Academy of Medical Sciences Cancer Institute and Hospital: Cancer Hospital Chinese Academy of Medical Sciences

\section{Yan Cai}

Chinese Academy of Medical Sciences Cancer Institute and Hospital: Cancer Hospital Chinese Academy of Medical Sciences

Xin Xu

Chinese Academy of Medical Sciences Cancer Institute and Hospital: Cancer Hospital Chinese Academy of Medical Sciences

Jia-Jie Hao

Chinese Academy of Medical Sciences Cancer Institute and Hospital: Cancer Hospital Chinese Academy of Medical Sciences

Ming-Rong Wang ( $\nabla$ wangmr2015@126.com )

Chinese Academy of Medical Sciences \& Peking Union Medical College

\section{Research}

Keywords: esophageal squamous cell carcinoma, metastasis, RNA binding protein, IGF2BP1, INHBA

Posted Date: September 21st, 2021

DOI: https://doi.org/10.21203/rs.3.rs-885735/v1

License: (c) (1) This work is licensed under a Creative Commons Attribution 4.0 International License. Read Full License 


\section{Abstract}

Background: The mechanisms underlying the occurrence and development of esophageal squamous cell carcinoma (ESCC) remains to be elucidated. The present study aims to investigate the roles and implications of IGF2BP1 overexpression in ESCC.

Methods: IGF2BP1 protein expression in ESCC samples was assessed by immunohistochemistry (IHC), and the mRNA abundance of IGF2BP1 and INHBA was analyzed with TCGA datasets and by RNA in situ hybridization (RISH). Cell viability, migration, invasion and in vivo metastasis assays were performed to explore the roles of IGF2BP1 overexpression in ESCC. RNA immunoprecipitation sequencing (RIP-seq) and mass spectrometry were applied to identify the target RNAs and interacting proteins of IGF2BP1, respectively. RIP-PCR, RNA pulldown, immunofluorescence (IF), gene-specific $m^{6} A$ PCR and RNA stability assays were used to uncover the molecular mechanisms underlying the malignant phenotypes of ESCC cells caused by IGF2BP1 dysregulation. The methylation level of the IGF2BP1 promoter region was detected by methylation-specific PCR (MSP-PCR). BTYNB, a small molecular inhibitor was evaluated for its inhibitory effect on the malignant phenotypes of ESCC cells.

Results: IGF2BP1 overexpression was detected in ESCC tissues and associated with the depth of tumor invasion. Knockdown of IGF2BP1 inhibited ESCC cell invasion and migration as well as tumor metastasis. Mechanistically, we observed that IGF2BP1 bound and stabilized INHBA mRNA and then enhanced the translation of INHBA, leading to the activation of Smad2/3 signaling, thus promoting malignant phenotypes. The mRNA level of INHBA was upregulated in ESCC tissues as well. Furthermore, IGF2BP1 interacted with G3BP stress granule assembly factor 1 (G3BP1) and activated the INHBA-Smad signaling. BTYNB abolished this activated signaling and significantly attenuated the malignant phenotypes of ESCC cells. In addition, IGF2BP1 mRNA expression in ESCC cells was negatively correlated with the level of its promoter methylation.

Conclusions: Elevated expression of IGF2BP1 is a frequent event in ESCC tissues and might be a candidate biomarker for the disease. IGF2BP1 overexpression promotes the invasion and migration of ESCC cells by activating the INHBA-TGF- $\beta$-Smad2/3 pathway, providing a potential therapeutic target for ESCC patients with high expression of IGF2BP1.

\section{Background}

Esophageal cancer is one of the major malignancies threatening human health. Esophageal squamous cell carcinoma (ESCC) accounts for more than $90 \%$ of esophageal cancer cases in China. Most patients with ESCC are diagnosed at an advanced stage, and the overall 5-year survival rate is only approximately $30 \%$ [1] due to invasive growth and distal metastasis. However, the molecular mechanisms underlying the invasion and metastasis of ESCC are still not fully understood, and there are no effective targeted drugs for clinical treatment to date. Therefore, there is an urgent need to identify the key molecules affecting the invasion and metastasis of ESCC. 
Insulin-like growth factor 2 mRNA binding protein 1 (IGF2BP1) is a highly conserved RNA binding protein that mainly binds mRNA and thereby affects RNA transcription, processing, translation and metabolism. IGF2BP1 overexpression is often correlated with poor prognosis in a variety of cancer types, including melanoma [2], breast [3], ovarian [4-6], colon [7, 8], liver [9, 10], and lung [11, 12] cancers. It has been reported that IGF2BP1 promotes cell proliferation, invasion and resistance to chemotherapeutic drugs and inhibitors by binding and stabilizing CD44, c-myc, ACTB and other oncogenic transcripts [13]. Moreover, IGF2BP1 has been proven to be a N6-methyladenosine $\left(\mathrm{m}^{6} \mathrm{~A}\right)$ reader that recognizes and binds $\mathrm{m}^{6} \mathrm{~A}$-modified mRNA and thus enhances its stability $[14,15]$. Additionally, IGF2BP1 has been designated an oncofetal protein due to its space-time specific expression pattern: it is predominantly expressed in embryonic development and suppressed in most adult tissues but re-expressed in multiple tumor types [16]. Several studies have reported that BTYNB, a selective inhibitor, can impair cell proliferation in vitro by blocking IGF2BP1 binding to its oncogenic transcripts $[17,18]$. Altogether, these studies suggest that IGF2BP1 might be a promising biomarker and therapeutic target.

We found remarkable upregulation of IGF2BP1 in ESCC tissues by immunohistochemistry (IHC). However, there is no information available on the role of IGF2BP1 in ESCC. In this study, we focused on the roles of IGF2BP1 overexpression in malignant phenotypes and the underlying mechanisms in ESCC cells, aiming to explore the possibility of IGF2BP1 as a biomarker and therapeutic target for the disease.

\section{Materials And Methods}

\section{Tissue specimens and cell lines}

ESCC and operative margin tissues from a total of 311 patients were procured from surgical resection specimens. All of the specimens were residual specimens collected after sampling for pathological diagnosis. All of the patients received no treatment prior to surgery and signed separate informed consent forms for sample collection and molecular analysis. The study was approved by the Ethics Committee of the Cancer Institute (Hospital), CAMS \& PUMC (No. 16-084/1163).

ESCC cell lines KYSE30, KYSE70, KYSE150, KYSE180, KYSE450 and KYSE510 were generously provided by Prof. Shimada (Kyoto University, Japan); TE1, TE4 and TE10 were purchased from ATCC, and Eca109 was purchased from Cell Resource Center, Institute of Basic Medicine, Chinese Academy of Medical Sciences. All of the cell lines were authenticated by short tandem repeat (STR) profiling and cultured in RPMI-1640 media with $10 \%$ fetal bovine serum (FBS500-S, AusGeneX) in a humidified incubator at $37^{\circ} \mathrm{C}$ and $5 \% \mathrm{CO}_{2}$.

\section{Immunohistochemistry (IHC)}

IHC assays were conducted as reported previously [19]. Slides were incubated with primary antibody IGF2BP1 (ab184305, 1:1000, Abcam) at $4^{\circ} \mathrm{C}$ overnight. After washing with PBS, the slides were incubated with a Mouse/Rabbit Enhanced Polymer Detection System (PV-9000, ZSGB-BIO), and the immunoreaction was visualized with DAB (ZLI-9017, ZSGB-BIO). The slides were scanned with a Nano 
Zoomer digital pathology biopsy scanner (HAMAMATSU, Japan). Immunoreactive scores were calculated by multiplying the scores of staining intensity and the percentage of positive cells. The intensity was scored as follows: 0 (negative), 1 (weak), 2 (moderate), and 3 (strong); the proportion of positive cells was scored as follows: 0 (negative), 1 (1\%-20\%), 2 (21\%-50\%), and 3 (51\%-100\%).

\section{RNA in situ hybridization (RISH)}

INHBA mRNA in situ hybridization was performed on $6 \mu \mathrm{m}$ thick tissue microarrays (TMAs) with RNAscope 2.5 HD Reagent Kit-BROWN (322300, ACD) following the manufacturer's instructions.

\section{Plasmids and cloning}

Cloning strategies including plasmids, primers used for PCR, and oligonucleotides are summarized in Additional file 1: Table S1-2. All constructs were validated by sequencing.

\section{Transfection and lentiviral transduction}

Cells were transfected with siRNA (GenePharma, China) for 48 hours or overexpression plasmids for 24 hours using Lipofectamine 3000 Transfection Reagent (L3000015, Thermo) according to the manufacturer's instructions. Lentiviruses expressing IGF2BP1-shRNA (shIGF2BP1) or no-silencing-shRNA (shNS) as a negative control were used to infect ESCC cells, and stable cell strains were selected by puromycin $(1 \mu \mathrm{g} / \mathrm{mL}$, Gibco) for one week. The siRNA or shRNA sequences are provided in Additional file 1: Table S2-3.

\section{Western blotting}

Total protein was isolated using RIPA buffer (C1053, Applygen) supplemented with protease inhibitors (B14001, Bimake) and phosphatase inhibitors (B15001, Bimake) and quantified with a Pierce BCA Protein Assay Kit (23225, Thermo). Primary antibodies for immunoblotting included IGF2BP1 (ab184305, 1:1,000, Abcam), INHBA (ab128958, 1:1,000, Abcam), Smad2/3 (8685T, 1:1,000, CST), G3BP1 (ab181150, 1:1,000, Abcam), p-Smad2 (s255, ab188334, 1:2,000, Abcam), METTL3 (ab195352, 1:1,000, Abcam), and METTL14 (26158-1-AP, 1:500, Proteintech). GAPDH (60004-1-lg, Proteintech) was used as a loading control. Secondary antibodies were used at a 1:5,000 dilution (goat anti-mouse/rabbit IgG, HRP conjugate, SA00001-1/2, Proteintech). The signals were detected with superenhanced chemiluminescence (ECL) detection reagent (29050, Engreen).

\section{Coimmunoprecipitation-based mass spectrometry (Co-IP- MS)}

Nondenaturing lysis buffer (P0013, Beyotime) was employed to isolate the total protein lysate. One microgram of IP antibody or rabbit IgG (B900610, Proteintech) was used to immunoprecipitate $1 \mu \mathrm{g}$ of total protein overnight at $4^{\circ} \mathrm{C}$ with rotation. Subsequently, $50 \mu \mathrm{L}$ of protein $\mathrm{A} / \mathrm{G}$ magnetic beads (MedChemExpress) were introduced into the mixture and incubated with rotation for $2 \mathrm{~h}$ at $4^{\circ} \mathrm{C}$. The beads were rinsed four times with washing buffer, after which they were eluted in $20 \mu \mathrm{L}$ of $1 \times$ loading buffer and denatured at $100^{\circ} \mathrm{C}$ for $10 \mathrm{~min}$. Protein samples were then subjected to WB assay or SDS- 
PAGE followed by Coomassie staining. Gel pieces were cut off and sent to Shanghai Applied Protein Technology Co. Ltd. for mass spectrometry analysis.

Gel pieces were digested overnight in $12.5 \mathrm{ng} / \mu \mathrm{L}$ trypsin. LC-MS/MS analysis was performed on a Q Exactive mass spectrometer (Thermo Scientific) that was coupled to Easy nLC (Thermo Fisher Scientific). MS/MS spectra were searched using the MASCOT engine (Matrix Science, London, UK; version 2.2) embedded in Proteome Discoverer 1.4 (Thermo Electron, San Jose, CA.) against the UniProt Human database (133549 sequences, download on March 3rd, 2013) and the decoy database. The cutoff of the global false discovery rate (FDR) for peptide and protein identification was set to 0.01 .

\section{Cell viability and colony formation assay}

A total of $1 \times 10^{3}$ cells were seeded into each well of a 96-well plate (with 3 replicates in each group), and the cell viability was quantified every $24 \mathrm{~h}$ using Cell Counting Kit-8 (CK-04, Dojindo, Japan) according to the manufacturer's instructions. Absorbance at $450 \mathrm{~nm}$ was measured by an Elx 808 Microplate Reader (BioTek, USA). For the colony formation assay, $1 \times 10^{3}$ cells were seeded into each well of a 6-well plate and treated with the indicated dose of BTYNB (with 3 replicates in each group) for 7-14 days. The colonies of cells were fixed with $100 \%$ methanol, stained using $0.1 \%$ crystal violet, and then counted.

\section{Cell apoptosis analysis}

Cells treated with $10 \mu \mathrm{M}$ BTYNB for $48 \mathrm{~h}$ were digested, collected and stained with fluorescently labeled Annexin V and PI using an Annexin V FITC Apoptosis Detection Kit (AD10, Dojindo). Flow cytometry was adopted to detect the percentage of apoptotic cells.

\section{Wound-healing assay}

Cells were seeded in six-well plates and grown until they reached full confluence. Ten microliter tips were used to scratch a wound vertically, and the scraped cells were removed by washing with PBS. The scratches were observed and photographed at 0,24 , and $48 \mathrm{~h}$ after being created. The scratch areas were measured using ImageJ (Ver. 1.51j8, NIH, USA).

\section{Cellular migration and invasion assays}

24-well Transwell plates with $8 \mu \mathrm{m}$ pores polycarbonate membrane inserts (3422, Corning, USA) were employed to assess the migration and invasion ability of ESCC cells. Matrigel basement membrane matrix (356234, Corning) was thawed on ice at $4^{\circ} \mathrm{C}$ overnight, and diluted in serum-free medium at a ratio of $1: 33(\mathrm{v} / \mathrm{v})$. Then $50 \mu \mathrm{L}$ of the diluted Matrigel matrix was added to the center of each Transwell insert for invasion assays. The plates were incubated at $37^{\circ} \mathrm{C}$ for 1 hour to allow the Matrigel matrix to form a gel. For migration assays, the Transwell inserts were not coated with Matrigel matrix.

Cultured cells were trypsinized and seeded into the upper chambers at a density of $1 \times 10^{5} /$ well in $200 \mu \mathrm{L}$ of serum-free medium, and $700 \mu \mathrm{L}$ of RPIM-1640 medium with $30 \%$ fetal bovine serum was added to the lower chambers as a chemoattractant. After incubation for 36 hours (KYSE30) or 24 hours (TE1) at $37^{\circ} \mathrm{C}$, 
the Transwell inserts were washed twice with PBS, fixed with methanol and acetone fixation solution (1:1, $\mathrm{v} / \mathrm{v}$ ), and stained with $0.1 \%$ crystal violet (Sigma). The cells on the inside of the inserts were gently removed using moistened cotton swabs. The polycarbonate membranes with stained cells were cut off from the Transwell inserts, placed on slide and mounted with coverslip, followed by scanning and imaging with a Nano Zoomer digital pathology biopsy scanner (HAMAMATSU, Japan). The areas covered by stained cells in three random fields were measured by ImageJ.

\section{Immunofluorescence (IF) staining}

Cells were fixed in $4 \%$ paraformaldehyde, permeabilized with $0.5 \%$ Triton $X-100$, blocked with $5 \%$ BSA and incubated with IGF2BP1 (sc-166344, 1:50, Santa) and G3BP1 (ab181150, 1:100, Abcam) antibodies at $4^{\circ} \mathrm{C}$ overnight. Then, the cells were incubated with fluorescent secondary antibody (rabbit IgG, CoraLite488 conjugate or mouse IgG, CoraLite594 conjugate, 1:200, Proteintech) for $1 \mathrm{~h}$ at room temperature in the dark, followed by staining with DAPI (ZLI-9557, ZSGB-BIO) for 5 min. Immunofluorescence was detected by confocal microscopy (PE double spinning disk confocal, USA).

\section{Xenograft assay}

Four-week-old female BALB/c nude mice (HFK Bioscience Co., LTD, Beijing, China) were purchased and randomly divided into two groups by body weight (10 per group). The mice were injected with $1 \times 10^{6}$ KYSE30 cells stably expressing shIGF2BP1 or shNon-silencing (shNS) via the tail vein. Eight weeks later, the mice were sacrificed, and the whole lung tissues were separated and fixed in Bouin's Fixative Solution (PH0976, Phygene). Then, the number of lung metastases was counted, and the lung tissues were embedded in paraffin, cut into $3 \mu \mathrm{m}$ sections, and stained with hematoxylin and eosin (H\&E).

All animal experiments were approved by the Animal Center of the Institute of National Cancer Center/Cancer Hospital, CAMS \& PUMC (NCC2019A016).

\section{Quantitative and Semiquantitative RT-PCR}

Total RNA was isolated using an RNApure Tissue \& Cell Kit (CW0506, Cwbiotech) following the manufacturer's instructions, and cDNA was synthesized using a HiFiScript cDNA Synthesis Kit (CW2569M, Cwbiotech). Then, quantitative real-time PCR was performed using a TB Green ${ }^{\text {TM }}$ Premix Ex Taq Kit (RR420A, TaKaRa) on an ABI QuantStudio DX real-time PCR system (ABI, USA). The relative expression levels of mRNA were assessed through the comparative threshold cycle method $\left(2^{-\triangle \Delta C t}\right)$ with GAPDH as an internal control. RT-PCR was conducted with TaKaRa Ex Taq (RR001A, TaKaRa) on a SimpliAmp Thermal Cycler (ABI, USA). All primers used in this study are listed in Additional file 1: Table S4.

\section{RNA coimmunoprecipitation combined with high- throughput sequencing (RIP-seq)}

RIP was performed using an EZ-Magna RIP Kit (17-701, Millipore). Briefly, $2 \times 10^{7}$ cells were harvested and resuspended in complete RIP lysis buffer (RIP lysis buffer supplemented with protease inhibitor 
cocktail and RNase inhibitor) on ice. One tenth of the supernatant of RIP lysate was saved as input. Five micrograms of IGF2BP1 antibody (RN007P, MBL) or negative control normal rabbit IgG (PP64B, Millipore) was conjugated to protein $A / G$ magnetic beads (MedChemExpress), followed by incubation with the RIP lysate in RIP immunoprecipitation buffer (RIP wash buffer supplemented with $0.5 \mathrm{M}$ EDTA and RNase inhibitor) for $4 \mathrm{~h}$ at $4^{\circ} \mathrm{C}$. Then, the immunoprecipitate was digested by proteinase $\mathrm{K}$ buffer. RNA was finally purified with TRIzol reagent (Invitrogen) and analyzed by qPCR or RNA-seq (Wuhan Seqhealth Tech Co. Ltd.). The sequences of primers for qPCR are described in Additional file 1: Table S4.

\section{Biotin RNA pull-down assay}

Cell lysates were prepared in pulldown buffer $(150 \mathrm{mM} \mathrm{KCl}, 0.5 \% \mathrm{NP}-40,25 \mathrm{mM}$ Tris-HCl pH 7.4, $0.5 \mathrm{mM}$ DTT, supplemented with proteasome inhibitors and $80 \mathrm{U} / \mathrm{mL}$ RNase inhibitors) and incubated with biotinlabeled DNA probes at $4^{\circ} \mathrm{C}$ for $2 \mathrm{~h}$ with rotation. Then, the RNA-protein complex was immunoprecipitated with streptavidin magnetic beads (MedChemExpress) after an additional $2 \mathrm{~h}$ of incubation at $4^{\circ} \mathrm{C}$ with rotation. The complex was divided into two equal portions. One was added to TRIzol reagent for RNA isolation followed by RT-PCR analysis; the other was eluted with $1 \times$ protein loading buffer for Western blotting. One fiftieth of the cell lysate was saved as input for RT-PCR and WB analysis. The sequences of biotin-labeled DNA probes against INHBA are provided in Additional file 1: Table S5.

\section{RNA stability assay}

ESCC cells stably expressing shIGF2BP1 or shNS were seeded into 12-well plates to $80 \%$ confluency after $24 \mathrm{~h}$. Then, the cells were treated with actinomycin $D(A c t D, 5 \mu \mathrm{g} / \mathrm{mL}$ ) for 0,2 , or $4 \mathrm{~h}$. Total RNA was extracted, and the relative level of INHBA mRNA at each time point was analyzed by quantitative real-time PCR with GAPDH as an internal control. The mRNA half-life was estimated according to a previous description [20].

\section{Gene-specific $\mathrm{m}^{6} \mathrm{~A}$ qPCR}

One hundred micrograms of total RNA was extracted, and one tenth of the RNA was saved as an input control. Protein $A / G$ magnetic beads (MCE) were prewashed and incubated with $5 \mu \mathrm{g}$ of anti- $\mathrm{m}^{6} \mathrm{~A}$ antibody (ABE572, Millipore) or rabbit IgG (PP64B, Millipore) for $2 \mathrm{~h}$ at $4^{\circ} \mathrm{C}$ with rotation. Then, the antibody-conjugated beads were mixed with total RNA in $1 \times$ binding buffer supplemented with RNase inhibitors for an additional $2 \mathrm{~h}$ at $4^{\circ} \mathrm{C}$ with rotation. The methylated mRNAs were eluted with elution buffer $(10 \mathrm{~mL}$ of $0.1 \mathrm{M} \mathrm{DTT}, 0.44 \mathrm{~g}$ of $\mathrm{NaCl}, 2.5 \mathrm{~mL}$ of $\mathrm{pH} 7.51 \mathrm{M}$ Tris- $\mathrm{HCl}, 0.1 \mathrm{~mL}$ of $0.5 \mathrm{M}$ EDTA, $0.5 \mathrm{~mL}$ of $10 \% \mathrm{SDS}, 10 \mu \mathrm{L}$ of RNase inhibitor, $\mathrm{ddH}_{2} \mathrm{O}$ up to $50 \mathrm{~mL}$ ) and recovered with the RNeasy Micro Kit (74004, Qiagen), further analyzed by RT-PCR along with input control.

\section{Methylation-specific PCR (MSP-PCR)}

Genomic DNA of ESCC cells was extracted using the QIAamp DNA Mini Kit (QIAGEN) and transformed with the Epitect Fast DNA Bisulfite Kit (QIAGEN). PCR amplification was performed with methylated primers (M primer) and unmethylated primers (U primer) by using the transformed DNA as a template. The sequences of $\mathrm{M}$ and $\mathrm{U}$ primer pairs against the first intron are provided in Additional file 1: Table S6. 


\section{Database analysis}

The Cancer Genome Atlas (TCGA) (https://cancergenome.nih.gov) datasets were employed to explore the mRNA expression of IGF2BP1 and INHBA in ESCC and nonmalignant tissues; the Wanderer interactive viewer (http://maplab.imppc.org/wanderer/) was used to analyze IGF2BP1 and INHBA mRNA expression in head and neck squamous cell carcinoma (HNSCC), lung squamous cell carcinoma (LUSC), cervical squamous cell carcinoma (CESC) and corresponding normal tissues. The expression of IGF2BP1 mRNA in all major tissues and organs in the human body was analyzed with the HPA database (https://www.proteinatlas.org/).

\section{Statistical analysis}

IBM SPSS Statistics 23.0 software was applied for data analysis, and $P<0.05$ was considered statistically significant. Fisher's exact test was used to assess the IHC score difference between ESCC tissues and adjacent noncancerous specimens. The correlation between the protein expression level and clinicopathological parameters was analyzed by Pearson's chi-square test. Comparisons between two groups were performed by independent samples T tests, and one-way ANOVA was used for multiple comparisons. RStudio software (1.1463) was used for Gene Ontology (GO) and pathway enrichment analysis.

Additional Materials and Methods are described in Additional files.

\section{Results}

\section{IGF2BP1 is highly expressed in ESCC tissues and is associated with the depth of tumor invasion}

We examined the protein expression level of IGF2BP1 in 311 ESCC tissues and 9 adjacent normal tissues by IHC. The results showed that IGF2BP1 was highly expressed in ESCC tissues (155/311, 49.8\%) but was not expressed or only weakly expressed in normal esophageal epithelia (Fig. 1A, Table 1). Positive staining was predominant in the cytoplasm of ESCC cells. A higher IGF2BP1 expression level was positively correlated with the depth of tumor invasion $\left(T_{1-2}\right.$ versus $\left.T_{3-4}\right)$, but no significant differences were found in other clinicopathological features, such as sex, age, histologic grade, lymph node metastasis and clinical stage (Table 1).

Furthermore, an analysis of RNA-seq data obtained from the TCGA database revealed that the mRNA expression level of IGF2BP1 was elevated in ESCC specimens compared with normal tissues (Fig. 1B), which was consistent with the $\mathrm{IHC}$ results.

\section{IGF2BP1 promotes ESCC cell migration, invasion and metastasis}

To identify the role of IGF2BP1 in ESCC, the expression of IGF2BP1 in 11 ESCC cell lines was detected by Western blotting. We found that IGF2BP1 had higher expression in KYSE30, KYSE450, KYSE140 and TE1 cells but was almost undetectable in KYSE70, KYSE150 and TE10 cells (Fig. 2A). KYSE30 and TE1 were 
applied for functional study as cell models. After transient knockdown of IGF2BP1 mediated by small interfering RNA (siRNA), there was no significant change in cell growth within six days compared with the negative control group (Fig. 2B). However, the migration and invasion ability of both cell lines were substantially repressed after IGF2BP1 silencing in the Transwell assays (Fig. 2C) and wound healing assays (Fig. 2D).

Next, KYSE30 cells stably expressing shRNA of IGF2BP1 (shIGF2BP1) or nonsilencing shRNA (shNS) conducted by lentivirus infection were injected into nude mice via the tail vein. The formation of lung metastatic tumors was observed in both groups six weeks later, but the number of lung metastases in the shIGF2BP1 group was significantly reduced, and the metastatic nodules were noticeably smaller (Fig. $2 \mathrm{E})$.

\section{IGF2BP1 increases INHBA mRNA stability, promoting ESCC cell migration and invasion}

Given that IGF2BP1 is an RNA binding protein, we performed RIP-seq in KYSE30 cells to identify its potential RNA targets. RIP-seq profiling revealed that most of the IGF2BP1-binding sites were highly enriched in coding regions (CDSs) of its mRNA targets, indicating that IGF2BP1 mainly regulates gene expression by binding to the CDS (Additional file 2: Figure S1). Through GO analysis of the identified results ( $\log F C>1$ and FDR < 0.01) by RIP-seq (Additional file 3: Table S7), we selected 30 genes related to cell migration and invasion as candidates (Additional file 4: Table S8). Then, quantitative real-time PCR was employed to detect the mRNA expression of the candidate genes in IGF2BP1 stable knockdown KYSE30 and TE1 cell lines. Notably, the reduction in INHBA mRNA abundance after IGF2BP1 knockdown was most significant among the 30 candidate genes in KYSE30 and TE1 cells (Fig. 3A, Additional file 5: Table S9). RIP-PCR and RNA pulldown using biotin-labeled DNA probes against INHBA mRNA further confirmed the interaction between IGF2BP1 protein and INHBA mRNA (Fig. 3B-C). Furthermore, silencing IGF2BP1 downregulated the protein expression of INHBA and Smad2/3 in KYSE30 and TE1 cells (Fig. 3D).

Previous studies have shown that IGF2BP1 can recognize N6-methyladenosine $\left(m^{6} A\right)$ and enhance mRNA stability and translation in an $\mathrm{m}^{6} \mathrm{~A}$-dependent manner $[14,15]$. Therefore, it is highly possible that IGF2BP1 binds and stabilizes the mRNA of INHBA. RNA stability assays showed that the half-life of INHBA mRNA was significantly shortened after knockdown of IGF2BP1 in KYSE30 and TE1 cells (Fig. $3 E)$. As the $m^{6} A$ writers METTL3 or METTL14 was repressed by siRNAs, the INHBA protein levels were decreased in both cell lines without affecting IGF2BP1 (Fig. 3F). Moreover, gene-specific $\mathrm{m}^{6} \mathrm{~A}$ qPCR further confirmed that INHBA was regulated by $\mathrm{m}^{6} \mathrm{~A}$ modification (Fig. $3 \mathrm{G}$ ).

INHBA is a member of the transforming growth factor $\beta$ (TGF- $\beta$ ) superfamily, which is closely associated with tumor invasion and metastasis. We employed a Transwell assay to assess the effects of INHBA on invasive and migratory phenotypes in ESCC cells. We observed that cell invasion and migration were inhibited after knockdown of INHBA mediated by siRNA (Fig. 3H), while transient overexpression of INHBA in cells with stable knockdown of IGF2BP1 partially overcame this inhibition (Fig. 3I). Western 
blotting results showed that Smad2/3 expression was repressed by IGF2BP1 depletion in KYSE30 and TE1 cells, while the IGF2BP1 knockdown-induced Smad2/3 decrease was reversed by INHBA overexpression (Fig. 3J).

\section{IGF2BP1 activates INHBA-Smad2/3 signaling by recruiting G3BP1}

It has been reported that IGF2BPs interact with other RNA-binding proteins (RBPs) to regulate mRNA targets $[21,22]$. To further elucidate the functional mechanism of IGF2BP1 in ESCC cells, we adopted CoIP-MS to investigate interactive partners of IGF2BP1 (Additional file 6: Figure S2). A total of 227 potential proteins were identified (Additional file 7: Table S10), and 46\% of them participate in RNA regulation (Additional file 8: Table S11). GO and pathway enrichment analysis revealed that most of them were involved in RNA processing, localization, stability, metabolism, transport and RNA splicing (Fig. $4 A-B)$.

We selected RNA-binding proteins as candidates for validation according to the following criteria: 1) By GO analysis, the candidates are related to migration and invasion. 2) According to GeneCards database, the sub-cellular localization of candidates are in the cytoplasm, which is the same as IGF2BP1 (https://www.genecards.org/). Based on the above criteria, we got eight candidate proteins in which G3BP1 had the top Coverage and PSM values in the IP group (Additional file 9: Table S12). Thus, we selected G3BP1 for further study. Endogenous G3BP1 was immunoprecipitated by IGF2BP1 antibody using a Co-IP assay, and vice versa (Fig. 4C). Immunofluorescence images captured using confocal microscopy confirmed the cytoplasmic colocalization of G3BP1 and IGF2BP1 in KYSE30 and TE1 cells (Fig. 4D). Moreover, Western blotting results showed that silencing G3BP1 by siRNA led to a decrease in INHBA and Smad2/3 and a slight reduction in IGF2BP1 (Fig. 4E).

\section{INHBA is significantly upregulated in ESCC, HNSC and invasive breast cancer}

We then analyzed TCGA transcriptome sequencing datasets and observed that the mRNA of INHBA was also increased in ESCC tissues (Fig. 5A). RISH analysis of tissue microarrays further confirmed the RNAseq results. More importantly, INHBA mRNA was mainly distributed in the peripheral tumor cells of cancer nests and the stroma of ESCC tissues but was negative in the normal esophageal epithelia and stroma (Fig. 5B). In addition, in HNSC and invasive breast cancer, INHBA mRNA was significantly elevated based on TCGA dataset analysis (http://maplab.imppc.org/wanderer/) (Fig. 5C).

\section{The small molecule inhibitor BTYNB significantly inhibits the invasion, migration and proliferation of ESCC cells in vitro}

Currently, there are no inhibitors in clinical trials that directly target IGF2BP1. Previous studies reported that a small molecule drug, BTYNB, could inhibit the binding of IGF2BP1 to c-Myc mRNA [17, 23]. BTYNB also impairs cell proliferation in vitro by blocking $\beta$-TRCP1, E2F and other transcripts $[17$, 18]. Therefore, it is reasonable to speculate that BTYNB might interfere with the IGF2BP1-driven malignant phenotypes in ESCC cells. We tested BTYNB with a Transwell assay by adding the drug into 
the lower chamber and found that the migration and invasion of KYSE30 and TE1 cells were significantly inhibited at $36 \mathrm{~h}$ and $24 \mathrm{~h}$, respectively (Fig. 6A). Meanwhile, cell viability and colony formation were impaired in a dose-dependent manner (Fig. 6B-C). In addition, $48 \mathrm{~h}$ of exposure to BTYNB increased the number of apoptotic cells (Fig. 6D). Western blotting analysis showed that INHBA and Smad2/3 expression was decreased after BTYNB treatment (Fig. 6E).

\section{Elevated expression of IGF2BP1 in ESCC cells is associated with hypomethylation of its first intron}

We further explored possible dysregulation of IGF2BP1 in other types of SCCs and found higher IGF2BP1 mRNA in HNSCC, LUSC, and CESC than in the corresponding normal tissues (http://maplab.imppc.org/wanderer/) (Fig. 7A). Moreover, we observed a high degree of consistency between the mRNA and protein levels of IGF2BP1 in 10 ESCC cell lines (Fig. 7B). Interestingly, according to the HPA (Human Protein Atlas) database, IGF2BP1 was almost absent in normal esophageal tissues and other normal tissues except in the embryo and reproductive system (Fig. 7C). To uncover the mechanism of IGF2BP1 mRNA upregulation in ESCC cells, we examined the methylation status of this gene. Three pairs of primers (methylated and unmethylated primers) were designed for MSP-PCR to detect distinct CG sites in the first intron of IGF2BP1 in both high and low IGF2BP1 expression cell lines. The results showed that the IGF2BP1 gene was hypomethylated in cell lines with high IGF2BP1 expression but hypermethylated in cell lines with low IGF2BP1 expression, except for TE10 (Fig. 7D).

\section{Discussion}

The insulin-like growth factor-2 mRNA-binding protein family (IGF2BPs), composed of IGF2BP1, IGF2BP2 and IGF2BP3, has a crucial role in early embryonic development. IGF2BP1 and IGF2BP3 are oncofetal proteins because they are mostly silent in adult organs, except in the reproductive system $[13,16,24]$. In contrast, IGF2BP2 is the only expressive IGF2BP in most adult tissues. IGF2BP1 and IGF2BP3 are reexpressed in many types of tumors, and IGF2BP2 was also found to be excessively expressed in malignancies due to genomic amplification according to pancancer analysis with TCGA data. Growing evidence supports the pro-oncogenic roles of these RNA-binding proteins in cancer progression by influencing their RNA target fate [25].

However, few studies on IGF2BP1 have been reported in ESCC. Herein, we discovered remarkably high expression of IGF2BP1 at both the mRNA and protein levels, indicating transcriptional dysregulation in ESCC. More importantly, our functional and mechanistic investigations revealed that IGF2BP1 facilitated the migration, invasion and metastasis of ESCC cells by activating the INHBA-Smad2/3 cascade. INHBA, a member of the TGF- $\beta$ superfamily, has been reported to be overexpressed in multiple types of cancers, including ESCC, and associated with poor prognosis [26-31]. Consistently, our analyses of TCGA data and RISH on TMAs demonstrated the upregulation of INHBA at the transcriptional level in ESCC tissues. In addition, we found that INHBA mRNA was frequently overexpressed in HNSC and invasive breast cancer. Combined with the spatial distribution of INHBA mRNA in ESCC tissues, we speculate that INHBA may play an important role in cell invasion and migration. Although the roles of INHBA in cancer are 
controversial, the majority favor its oncogenic effects. Seder et al. reported that INHBA promoted cell proliferation and was regulated by promoter demethylation in ESCC cells [32]. In the present study, we identified INHBA as a direct target of IGF2BP1 with a functional role in tumor invasion and metastasis induced by IGF2BP1. Mechanistically, IGF2BP1 bound and stabilized INHBA mRNA, thus enhancing the translation of INHBA. Moreover, as an $\mathrm{m}^{6} \mathrm{~A}$ reader proven by recent research, IGF2BP1 preferentially recognizes $m^{6} \mathrm{~A}$-modified $m R N A s$ and promotes their stability in an $m^{6} \mathrm{~A}$-dependent manner [33]. We indeed observed that INHBA mRNA was $\mathrm{m}^{6} \mathrm{~A}$ modified and that the turnover of INHBA was $\mathrm{m}^{6} \mathrm{~A}$ dependent. Therefore, it is likely that mRNA methylation is required in the regulation of INHBA by IGF2BP1.

IGF2BP1 functions by interacting with other RNA-binding proteins. Together they participate in forming ribonucleoprotein (RNP) granules that regulate mRNA translation, localization, and turnover [21]. We identified 105 proteins related to RNA processing by MS, accounting for $46 \%$ of all interactive proteins of IGF2BP1 in KYSE30 cells. We found that G3BP1 was a partner of IGF2BP1 and contributed to positive regulation of INHBA-Smad2/3 signaling. G3BP1 contains two C-terminal motifs (associated with RNA binding) and an RNA recognition motif (RRM). It has been demonstrated that G3BP1 promotes stressinduced RNA granule interactions to preserve polyadenylated mRNA [34]. Meanwhile, G3BP1 is involved in protein degradation by stably associating with USP10 deubiquitinase [35]. Our experimental results showed that after G3BP1 knockdown, IGF2BP1 protein was slightly downregulated, while INHBA protein was significantly decreased. Therefore, G3BP1 might activate IGF2BP1-INHBA-Smad2/3 signaling by enhancing the mRNA stability of INHBA. The specific details regarding how G3BP1 mediates IGF2BP1 to elevate INHBA still need further investigation.

Several studies have demonstrated that BTYNB, a structure-specific inhibitor, could block the binding of IGF2BP1 to its oncogenic target mRNA, thus disrupting their interaction $[17,18]$. We evaluated the efficacy of BTYNB in vitro and found that the typical malignant phenotypes of ESCC cell lines with high IGF2BP1 expression were sharply repressed, and apoptotic cells were increased after BTYNB exposure. At the molecular level, BTYNB treatment led to attenuated IGF2BP1-INHBA-Smad2/3 signaling. As mentioned above, IGF2BP1 has long been considered an oncofetal protein. In fact, according to the Atlas report, IGF2BP1 mRNA is expressed only in the testis and placenta and weakly in the kidney, and IGF2BP1 protein is expressed only in the adult testis, ovary, and bronchial tissues

[https://www.proteinatlas.org/ENSG00000159217-IGF2BP1/tissue], supporting an expression of IGF2BP1 pattern in few adult tissues. In the present study, our immunohistochemistry results showed that IGF2BP1 is highly expressed in nearly $50 \%$ of ESCC tissues but not expressed or only weakly expressed in all surgical margin specimens. Based on these published data and our observations, IGF2BP1 could be a very promising target for ESCC, making it possible to specifically target tumor cells without disturbing noncancerous tissues. These data imply that IGF2BP1 is a potential molecular target for ESCC therapy.

Little information is available describing how the expression of IGF2BP1 is modulated at the transcriptional and posttranscriptional levels. It has been proposed that IGF2BP1 transcription is induced by $\beta$-catenin [36] and enhanced by c-Myc through a positive feedback loop [37]. In addition, let-7 could 
regulate IGF2BP1 posttranscriptionally [38]. Our observation linked genomic hypomethylation in the first intron to high IGF2BP1 expression, suggesting a new perspective on aberrant transcription of this gene.

\section{Conclusions}

Our data demonstrate that upregulation of IGF2BP1 is a frequent event in ESCC tissues and might serve as a candidate biomarker for the disease. The present study reveals for the first time that elevated IGF2BP1 plays a pivotal role in the invasion and migration of ESCC cells by activating the IHNBASmad2/3 signaling pathway, providing a promising and attractive target for ESCC patients with high expression of IGF2BP1.

\section{Abbreviations}

ESCC: Esophageal squamous cell carcinoma, SCC: Squamous cell carcinoma, HNSCC: Head and neck squamous cell carcinoma, LUSC: Lung squamous cell carcinoma, CESC: Cervical squamous cell carcinoma, IHC: Immunohistochemistry, RISH: RNA in situ hybridization, TMA: Tissue microarray, H\&E staining: Haematoxylin \& eosin staining, IF: Immunofluorescence, siRNA: small interfering RNA, NC: Negative control, RIP: RNA co-Immunoprecipitation, RT-PCR: Reverse transcription-polymerase chain reaction, WB: Western blotting, Co-IP-MS: Co-immunoprecipitation-mass spectrometry, MSP-PCR: Methylation specific PCR, TCGA: The Cancer Genome Atlas, HPA: Human Protein Atlas, GO: Gene Ontology, KEGG: Kyoto Encyclopedia of Genes and Genomes, RBPs: RNA-binding proteins, IGF2BPs: Insulin-like growth factor-2 mRNA-binding proteins, CDS: Coding sequence, $\mathrm{m}^{6} \mathrm{~A}$ : N6-methyladenosine, TGF- $\beta$ : Transforming growth factor $\beta$, G3BP1: GTPase activating protein (SH3 domain) binding protein 1 , RNP: Ribonucleoprotein, RRM: RNA recognition motif, ActD: actinomycin D.

\section{Declarations}

\section{Ethics approval and consent to participate}

The study was approved by the Ethics Committee of National Cancer Center/Cancer Institute (Hospital), CAMS \& PUMC (No. 16-084/1163). All the animal experiments were approved by the Animal Center of the Institute of National Cancer Center/Cancer Hospital, CAMS \& PUMC (NCC2019A016).

\section{Consent for publication}

Not applicable.

\section{Availability of data and materials}

The datasets (TCGA.ESCA. sampleMap/HiSeqV2) analyzed during the current study are available in the UCSC Xena TCGA hub repository, https://tcga.xenahubs.net. 
The authors declare that they have no competing interests.

\section{Funding}

This study was supported by National Key Research and Development Program of China (2017YFE0112100), National Natural Science Foundation of China (81930077), Chinese Academy of Medical Sciences (CAMS) Fundamental Research Funds for the Central Public-interest Scientific Institution (2018RC310008), and Potential Development Projects of Cancer Hospital, CAMS (PY2018B01).

\section{Authors' contributions}

MRW and JJH conceived and supervised the study. JJW contributed to the study design and performed most of the assays. DXC and JJW performed animal experiments. YC and JJW conducted immunohistochemistry assay. XX and YC provided necessary materials. JJW interpreted the data and drafted the manuscript. MRW and JJH revised the manuscript. All authors read and approved the final manuscript.

\section{Acknowledgements}

Not applicable.

\section{References}

1. Zeng H, Chen W, Zheng R, Zhang S, Ji JS, Zou X, et al. Changing cancer survival in China during 2003-15: a pooled analysis of 17 population-based cancer registries. Lancet Glob Health. 2018,6(5):e555-e67.

2. Elcheva I, Tarapore RS, Bhatia N, Spiegelman VS. Overexpression of mRNA-binding protein CRD-BP in malignant melanomas. Oncogene. 2008,27(37):5069-74.

3. Gu W, Katz Z, Wu B, Park HY, Li D, Lin S, et al. Regulation of local expression of cell adhesion and motility-related mRNAs in breast cancer cells by IMP1/ZBP1. J Cell Sci. 2012,125(Pt 1):81-91.

4. Bley N, Schott A, Muller S, Misiak D, Lederer M, Fuchs T, et al. IGF2BP1 is a targetable SRC/MAPKdependent driver of invasive growth in ovarian cancer. RNA Biol. 2021,18(3):391-403.

5. Kobel M, Weidensdorfer D, Reinke C, Lederer M, Schmitt WD, Zeng K, et al. Expression of the RNAbinding protein IMP1 correlates with poor prognosis in ovarian carcinoma. Oncogene. 2007,26(54):7584-9.

6. Fan L, Lin Y, Lei H, Shu G, He L, Yan Z, et al. A newly defined risk signature, consisting of three $m(6) A$ RNA methylation regulators, predicts the prognosis of ovarian cancer. Aging (Albany NY). 2020,12(18):18453-75.

7. Zhang XL, Li KJ, Feng JX, Liu GJ, Feng YL. Blocking the IGF2BP1-promoted glucose metabolism of colon cancer cells via direct de-stabilizing mRNA of the LDHA enhances anticancer effects. Mol Ther 
Nucleic Acids. 2021,23:835-46.

8. Dimitriadis E, Trangas T, Milatos S, Foukas PG, Gioulbasanis I, Courtis N, et al. Expression of oncofetal RNA-binding protein CRD-BP/IMP1 predicts clinical outcome in colon cancer. Int J Cancer. 2007,121(3):486-94.

9. Zhang J, Hu K, Yang YQ, Wang Y, Zheng YF, Jin Y, et al. LIN28B-AS1-IGF2BP1 binding promotes hepatocellular carcinoma cell progression. Cell Death Dis. 2020,11(9):741.

10. Hammerle M, Gutschner T, Uckelmann H, Ozgur S, Fiskin E, Gross M, et al. Posttranscriptional destabilization of the liver-specific long noncoding RNA HULC by the IGF2 mRNA-binding protein 1 (IGF2BP1). Hepatology. 2013,58(5):1703-12.

11. Kato T, Hayama S, Yamabuki T, Ishikawa N, Miyamoto M, Ito T, et al. Increased expression of insulinlike growth factor-II messenger RNA-binding protein 1 is associated with tumor progression in patients with lung cancer. Clin Cancer Res. 2007,13(2 Pt 1):434-42.

12. Zhang J, Luo W, Chi X, Zhang L, Ren Q, Wang H, et al. IGF2BP1 silencing inhibits proliferation and induces apoptosis of high glucose-induced non-small cell lung cancer cells by regulating Netrin-1. Arch Biochem Biophys. 2020,693:108581.

13. Huang X, Zhang H, Guo X, Zhu Z, Cai H, Kong X. Insulin-like growth factor 2 mRNA-binding protein 1 (IGF2BP1) in cancer. J Hematol Oncol. 2018,11(1):88.

14. Wang X, Lu Z, Gomez A, Hon GC, Yue Y, Han D, et al. N6-methyladenosine-dependent regulation of messenger RNA stability. Nature. 2014,505(7481):117-20.

15. Huang $H$, Weng $H$, Sun W, Qin X, Shi H, Wu H, et al. Recognition of RNA N(6)-methyladenosine by IGF2BP proteins enhances mRNA stability and translation. Nat Cell Biol. 2018,20(3):285-95.

16. Bell JL, Wachter K, Muhleck B, Pazaitis N, Kohn M, Lederer M, et al. Insulin-like growth factor 2 mRNA-binding proteins (IGF2BPs): post-transcriptional drivers of cancer progression? Cell Mol Life Sci. 2013,70(15):2657-75.

17. Mahapatra L, Andruska N, Mao C, Le J, Shapiro DJ. A Novel IMP1 Inhibitor, BTYNB, Targets c-Myc and Inhibits Melanoma and Ovarian Cancer Cell Proliferation. Transl Oncol. 2017,10(5):818-27.

18. Muller S, Bley N, Busch B, Glass M, Lederer M, Misiak C, et al. The oncofetal RNA-binding protein IGF2BP1 is a druggable, post-transcriptional super-enhancer of E2F-driven gene expression in cancer. Nucleic Acids Res. 2020,48(15):8576-90.

19. Shi F, Shang L, Pan BQ, Wang XM, Jiang YY, Hao JJ, et al. Calreticulin promotes migration and invasion of esophageal cancer cells by upregulating neuropilin-1 expression via STAT5A. Clin Cancer Res. 2014,20(23):6153-62.

20. Chen CY, Ezzeddine N, Shyu AB. Messenger RNA half-life measurements in mammalian cells. Methods Enzymol. 2008,448:335-57.

21. Jonson L, Vikesaa J, Krogh A, Nielsen LK, Hansen T, Borup R, et al. Molecular composition of IMP1 ribonucleoprotein granules. Mol Cell Proteomics. 2007,6(5):798-811. 
22. Jonson L, Christiansen J, Hansen TVO, Vikesa J, Yamamoto Y, Nielsen FC. IMP3 RNP safe houses prevent miRNA-directed HMGA2 mRNA decay in cancer and development. Cell Rep. 2014,7(2):53951.

23. Mahapatra L, Mao C, Andruska N, Zhang C, Shapiro DJ. High-throughput fluorescence anisotropy screen for inhibitors of the oncogenic mRNA binding protein, IMP-1. J Biomol Screen. 2014,19(3):427-36.

24. Lederer M, Bley N, Schleifer $C$, Huttelmaier $S$. The role of the oncofetal IGF2 mRNA-binding protein 3 (IGF2BP3) in cancer. Semin Cancer Biol. 2014,29:3-12.

25. Degrauwe N, Suva ML, Janiszewska M, Riggi N, Stamenkovic I. IMPs: an RNA-binding protein family that provides a link between stem cell maintenance in normal development and cancer. Genes Dev. 2016,30(22):2459-74.

26. Lyu S, Jiang $C, X u R$, Huang $Y$, Yan S. INHBA upregulation correlates with poorer prognosis in patients with esophageal squamous cell carcinoma. Cancer Manag Res. 2018,10:1585-96.

27. Bashir M, Damineni S, Mukherjee G, Kondaiah P. Activin-A signaling promotes epithelialmesenchymal transition, invasion, and metastatic growth of breast cancer. NPJ Breast Cancer. 2015,1:15007.

28. Okano M, Yamamoto H, Ohkuma H, Kano Y, Kim H, Nishikawa S, et al. Significance of INHBA expression in human colorectal cancer. Oncol Rep. 2013,30(6):2903-8.

29. Wang Q, Wen YG, Li DP, Xia J, Zhou CZ, Yan DW, et al. Upregulated INHBA expression is associated with poor survival in gastric cancer. Med Oncol. 2012,29(1):77-83.

30. Seder CW, Hartojo W, Lin L, Silvers AL, Wang Z, Thomas DG, et al. Upregulated INHBA expression may promote cell proliferation and is associated with poor survival in lung adenocarcinoma. Neoplasia. 2009,11(4):388-96.

31. Lee HY, Li CC, Huang CN, Li WM, Yeh HC, Ke HL, et al. INHBA overexpression indicates poor prognosis in urothelial carcinoma of urinary bladder and upper tract. J Surg Oncol. 2015,111(4):414-22.

32. Seder CW, Hartojo W, Lin L, Silvers AL, Wang Z, Thomas DG, et al. INHBA overexpression promotes cell proliferation and may be epigenetically regulated in esophageal adenocarcinoma. $J$ Thorac Oncol. 2009,4(4):455-62.

33. Huang $H$, Weng $H$, Sun W, Qin $X$, Shi H, Wu H, et al. Author Correction: Recognition of RNA N(6)methyladenosine by IGF2BP proteins enhances mRNA stability and translation. Nat Cell Biol. 2018,20(9):1098.

34. Aulas A, Caron G, Gkogkas CG, Mohamed NV, Destroismaisons L, Sonenberg N, et al. G3BP1 promotes stress-induced RNA granule interactions to preserve polyadenylated mRNA. J Cell Biol. 2015,209(1):73-84.

35. Meyer C, Garzia A, Morozov P, Molina H, Tuschl T. The G3BP1-Family-USP10 Deubiquitinase Complex Rescues Ubiquitinated 40S Subunits of Ribosomes Stalled in Translation from Lysosomal Degradation. Mol Cell. 2020,77(6):1193-205 e5. 
36. Noubissi FK, Elcheva I, Bhatia N, Shakoori A, Ougolkov A, Liu J, et al. CRD-BP mediates stabilization of betaTrCP1 and c-myc mRNA in response to beta-catenin signalling. Nature. 2006,441(7095):898901.

37. Noubissi FK, Nikiforov MA, Colburn N, Spiegelman VS. Transcriptional Regulation of CRD-BP by Cmyc: Implications for c-myc Functions. Genes Cancer. 2010,1(10):1074-82.

38. Boyerinas B, Park SM, Murmann AE, Gwin K, Montag AG, Zillhardt M, et al. Let-7 modulates acquired resistance of ovarian cancer to Taxanes via IMP-1-mediated stabilization of multidrug resistance 1. Int J Cancer. 2012,130(8):1787-97.

\section{Tables}

Table 1. The association between IGF2BP1 expression and the clinicopathological variables 


\begin{tabular}{|c|c|c|c|c|}
\hline \multirow[t]{2}{*}{ Clinicopathological parameter } & \multirow[t]{2}{*}{ Case number } & \multicolumn{3}{|c|}{ IGF2BP1 expression } \\
\hline & & Positive (\%) & $c^{2}$ & $P$-value \\
\hline \multicolumn{5}{|l|}{ age } \\
\hline$<60$ & 98 & $44(44.90)$ & \multirow[t]{2}{*}{0.361} & \multirow[t]{2}{*}{0.548} \\
\hline$\geq 60$ & 213 & $111(52.10)$ & & \\
\hline \multicolumn{5}{|l|}{ gender } \\
\hline male & 225 & $114(50.70)$ & \multirow[t]{2}{*}{0.223} & \multirow[t]{2}{*}{0.637} \\
\hline female & 86 & $41(47.70)$ & & \\
\hline \multicolumn{5}{|l|}{ Grade } \\
\hline G1 & 44 & $16(36.36)$ & \multirow[t]{3}{*}{3.724} & \multirow[t]{3}{*}{0.155} \\
\hline G2 & 200 & $104(52.00)$ & & \\
\hline G3 & 67 & $35(52.24)$ & & \\
\hline \multicolumn{5}{|l|}{ pT } \\
\hline T1-2 & 84 & $33(39.29)$ & \multirow[t]{2}{*}{5.127} & \multirow[t]{2}{*}{0.024} \\
\hline T3-4 & 227 & $122(53.74)$ & & \\
\hline \multicolumn{5}{|l|}{ pN } \\
\hline NO & 161 & $81(50.31)$ & \multirow[t]{2}{*}{0.03} & \multirow[t]{2}{*}{0.863} \\
\hline N1-4 & 150 & $74(49.33)$ & & \\
\hline \multicolumn{5}{|l|}{ Stage } \\
\hline Q & 30 & $14(46.67)$ & \multirow[t]{4}{*}{0.493} & \multirow[t]{4}{*}{0.92} \\
\hline Q & 143 & $71(49.65)$ & & \\
\hline Q & 120 & $62(51.67)$ & & \\
\hline 口 & 18 & $8(44.44)$ & & \\
\hline
\end{tabular}

Statistical significance $(P<0.05)$ is shown in bold.

Figures 

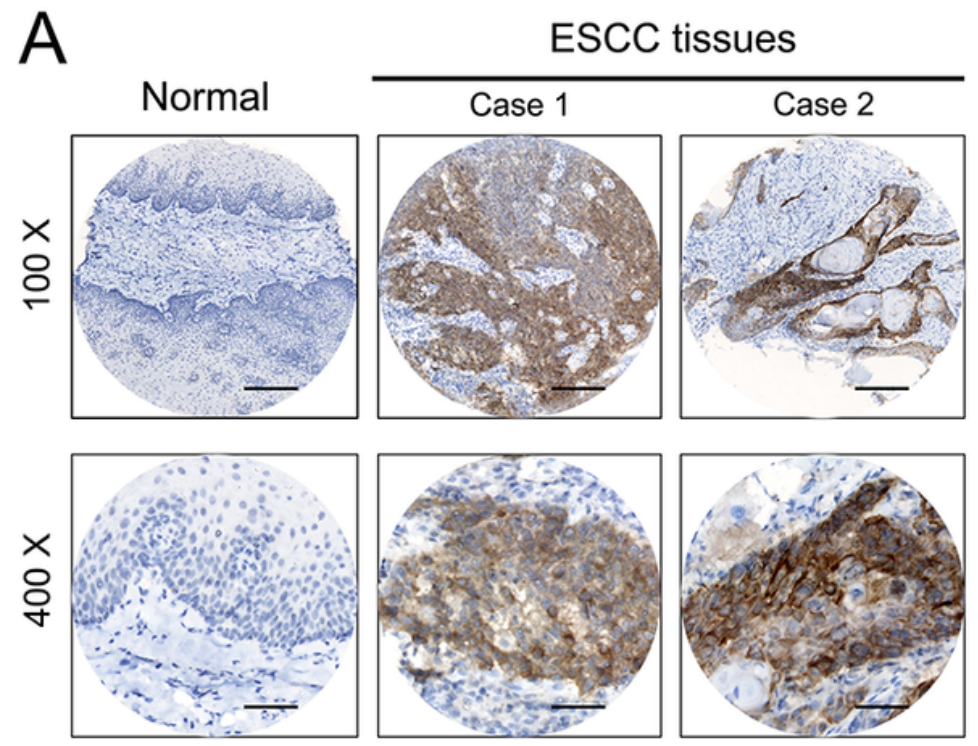

B

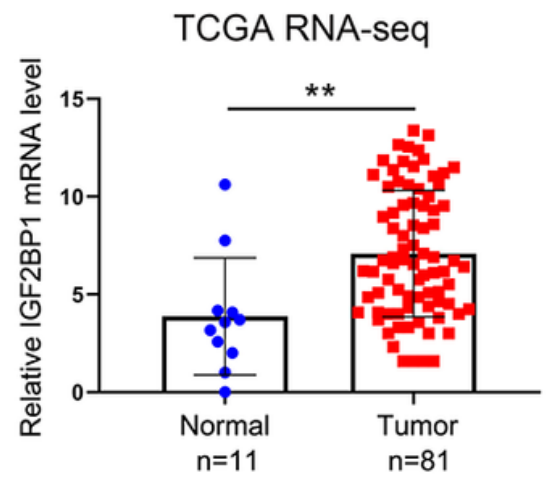

\section{Figure 1}

IGF2BP1 is overexpressed in ESCC tissues. A. Representative IHC staining of IGF2BP1 in ESCC and adjacent nonmalignant tissues. Scale bar $=200 \mu \mathrm{m}(100 \times)$, scale bar $=50 \mu \mathrm{m}(400 \times)$. B. IGF2BP1 mRNA levels in ESCC patients and normal tissues in TCGA database. ${ }^{*} \mathrm{P}<0.01$. 
A

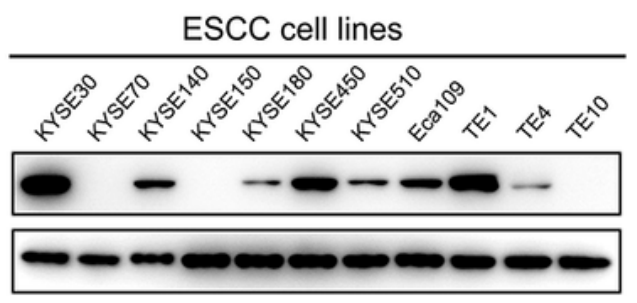

C
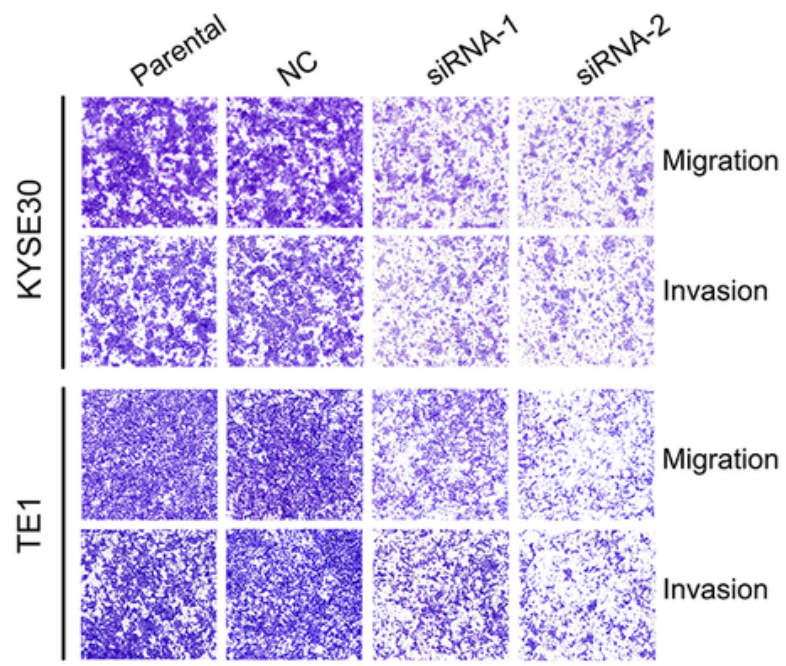
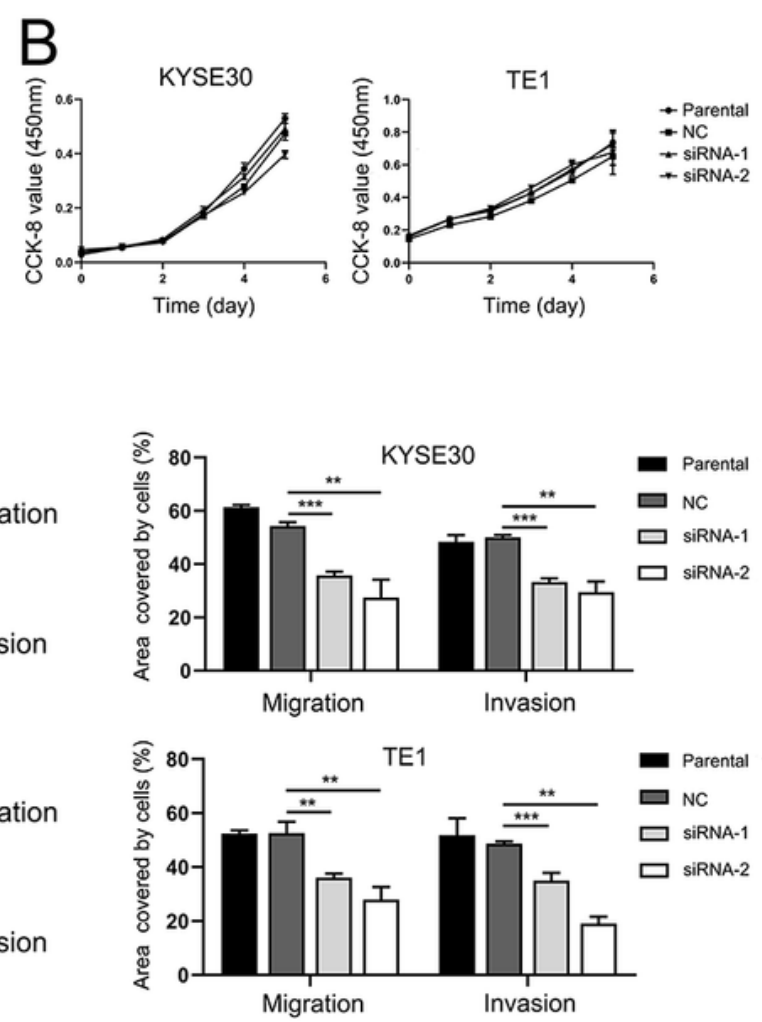
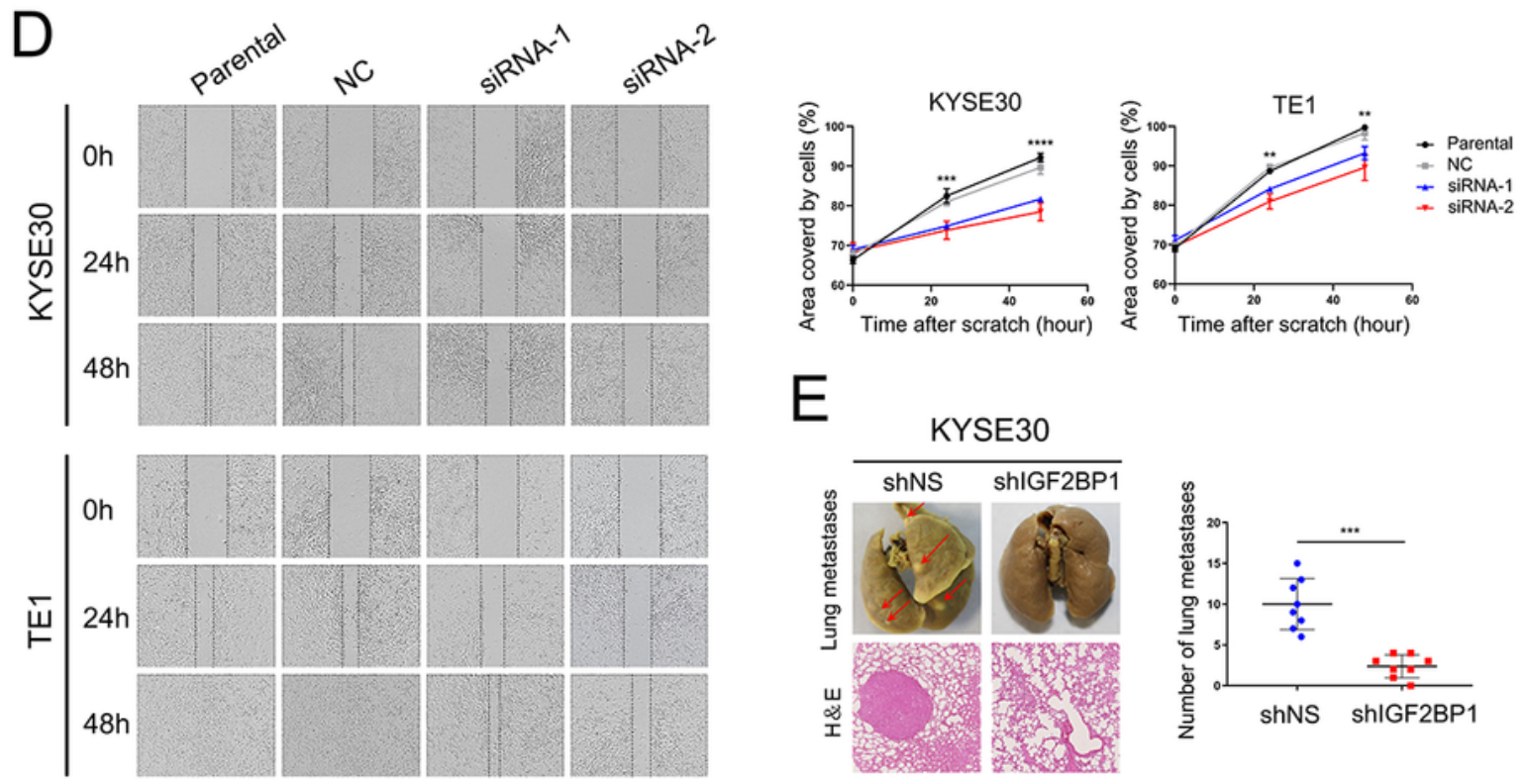

\section{Figure 2}

IGF2BP1 promotes the migration and invasion of ESCC cells in vitro as well as lung metastasis in vivo. A. Western blotting analysis of IGF2BP1 expression in ESCC cell lines. B. Cell viability was quantified using a CCK-8 assay. C. Cell migration and invasion abilities were examined using Transwell assays.

Representative results (left) and statistical plots (right) are shown. D. Cell motility was assessed by the wound-healing assay. Representative results (left) and statistical plots (right) are shown. E. Cell 
metastatic potential was evaluated using an in vivo pulmonary metastasis assay. Representative images of fixed lung tissues (top) and the results of H\&E staining (bottom) are shown. The arrows indicate the lung metastatic nodules. The number of metastatic nodules was plotted (right). ${ }^{\star \star} \mathrm{P}<0.01,{ }^{* \star} \mathrm{P}<0.001$,

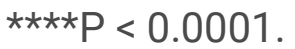

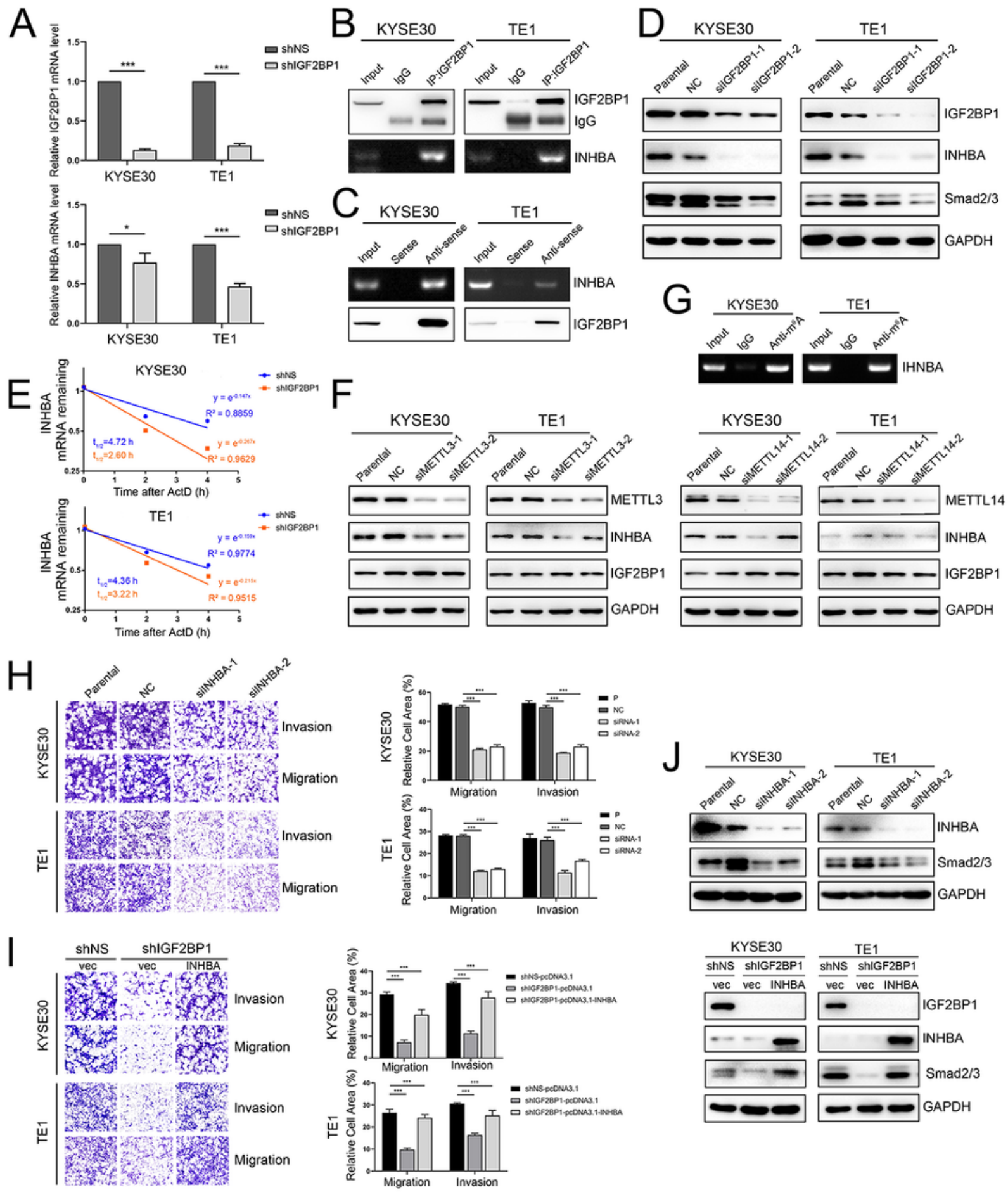

Figure 3 
IGF2BP1 enhances ESCC cell migration and invasion by activating INHBA-Smad2/3 signaling. A-B. The interaction between IGF2BP1 protein and INHBA mRNA in ESCC cells was validated with RIP-PCR (A) and RNA pull-down assay (B). IGF2BP1 protein enriched by antibody or coprecipitated by biotin-labeled DNA probe against INHBA mRNA was detected by Western blotting, INHBA mRNA enriched by biotinylated probe or coprecipitated by IGF2BP1 antibody was analyzed by RT-PCR. IgG antibody or biotin-labeled sense probes were used as negative controls. C. The mRNA levels of IGF2BP1 and INHBA in KYSE30 and TE1 cells after IGF2BP1 knockdown were determined by real-time PCR. D. Western blotting analysis of IGF2BP1, INHBA, and Smad2/3 in ESCC cells transfected with IGF2BP1-specific siRNA or NC siRNA. E. The decay rate of INHBA mRNA after IGF2BP1 depletion was evaluated by RNA stability assay. Relative mRNA expression at 0, 2, and $4 \mathrm{~h}$ after ActD treatment was analyzed by real-time PCR, and GAPDH was used as an internal control. The decay rate of mRNA was measured in terms of half-life time (t1/2). F. Western blotting analysis of IGF2BP1, INHBA, and METTL3/14 after METTL3/14 transient knockdown. G. m6A modification in INHBA mRNA was tested by gene-specific m6A PCR. Methylated INHBA mRNA in total RNA enriched by m6A antibody was analyzed by RT-PCR. IgG antibody served as negative control. $\mathrm{H}-\mathrm{I}$. Cell migration and invasion abilities were examined with Transwell assays. Representative results (left) and statistical plots (right) are shown. J. Western blotting analysis of IHNBA and Smad2/3 in ESCC cells transfected with INHBA siRNA (above) and in ESCC cells stably expressing shIGF2BP1 transfected with pcDNA3.1-INHBA or empty vector (below). ${ }^{\star} \mathrm{P}<0.05, * \star * \mathrm{P}<0.001$. 
A

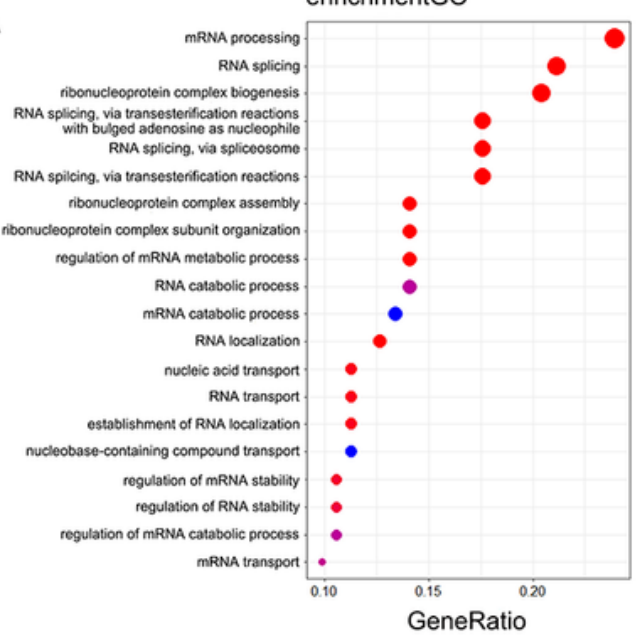

C
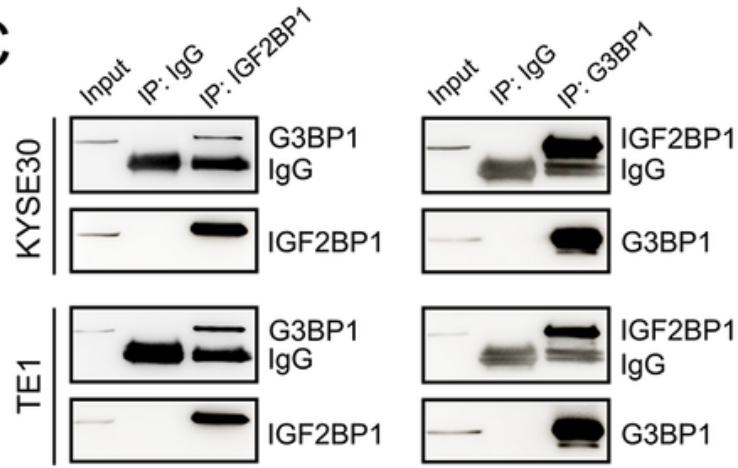

$E$

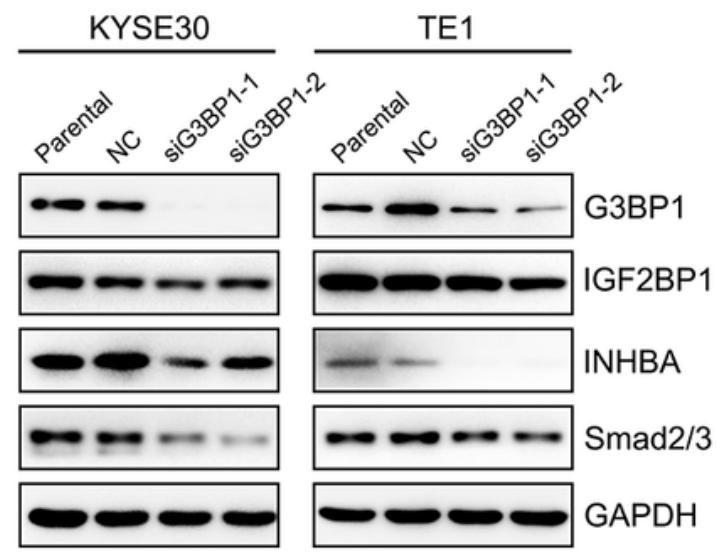

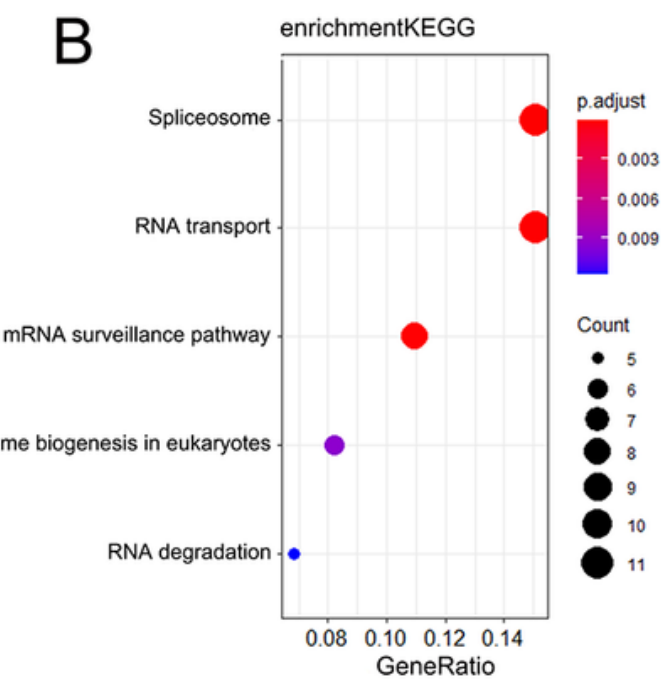

$\mathrm{D}$
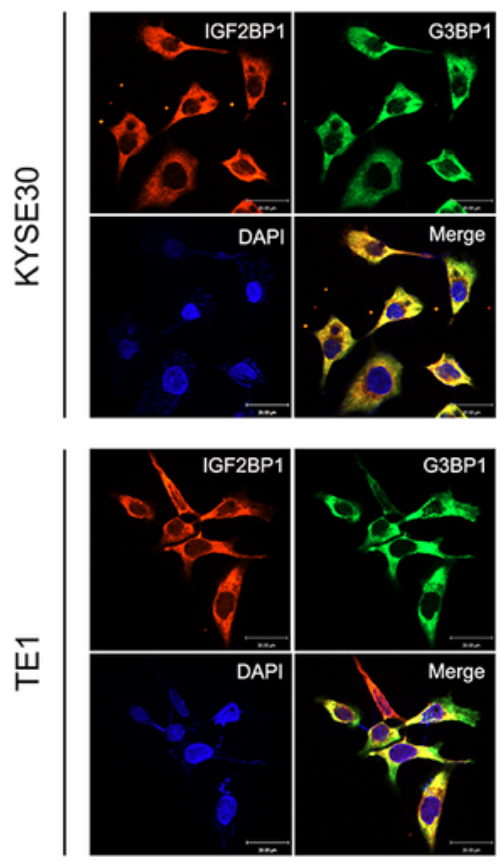

\section{Figure 4}

G3BP1 interacts with IGF2BP1 and facilitates the activation of INHBA-Smad2/3 signaling. A-B. Bubble plot of GO (A) and KEGG (B) enrichment based on mass spectrometry results. The alteration in color from red to blue illustrates a decrease in the $P$ value, and the dot size indicates the number of genes enriched in KEGG terms. C. The interaction between IGF2BP1 and G3BP1 in ESCC cells was detected with an endogenous immunoprecipitation assay. D. Cellular localization of endogenously expressed IGF2BP1 
(red) or G3BP1 (green) was detected by immunofluorescence staining using laser confocal microscopy. DAPI was used to stain nuclei (blue). Scale bar $=30 \mu \mathrm{m}$. E. Western blotting analysis of IGF2BP1, INHBA, and Smad2/3 in ESCC cells transfected with NC-siRNA or G3BP1 siRNA. GAPDH was used as a loading control.
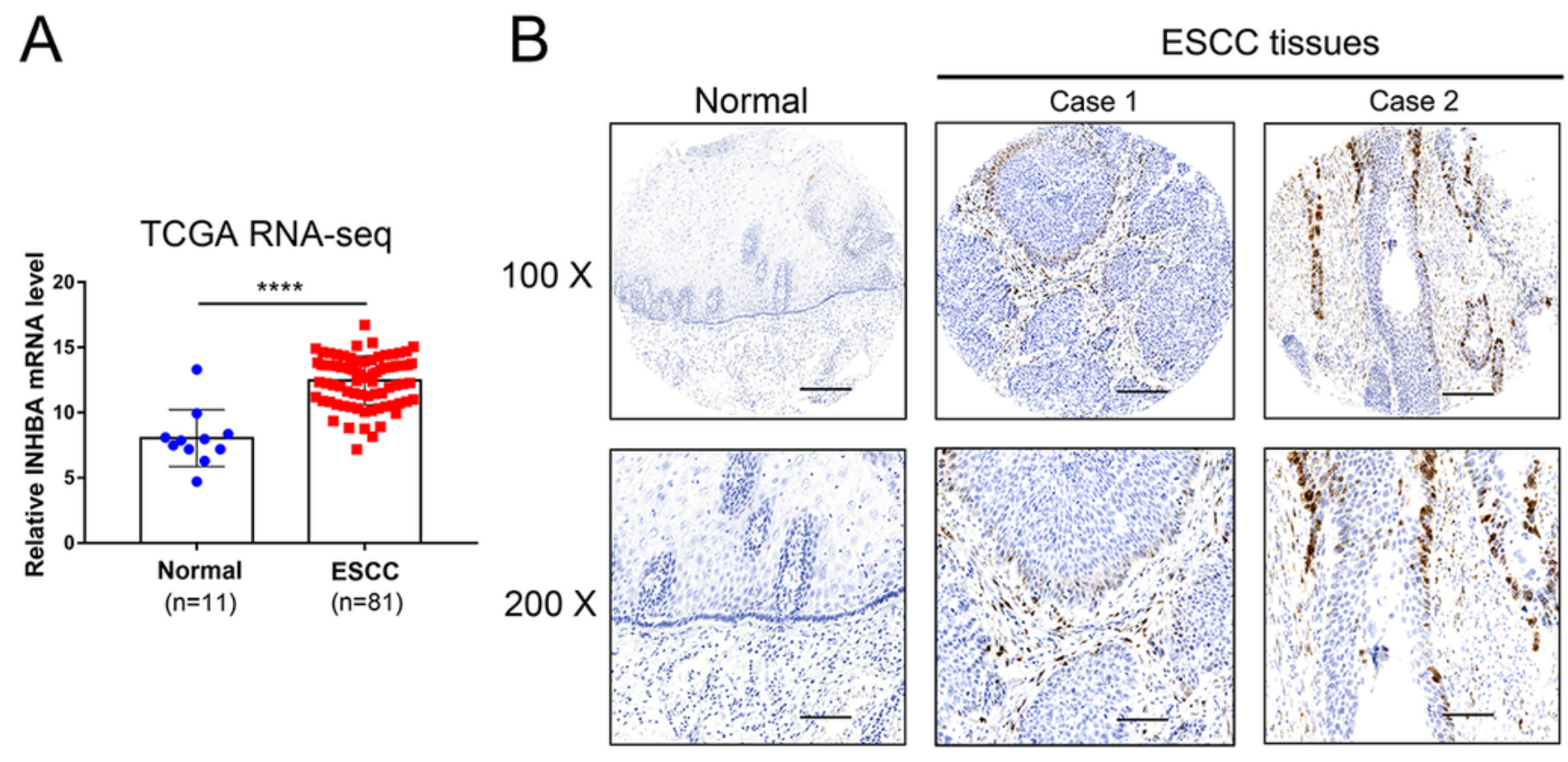

C

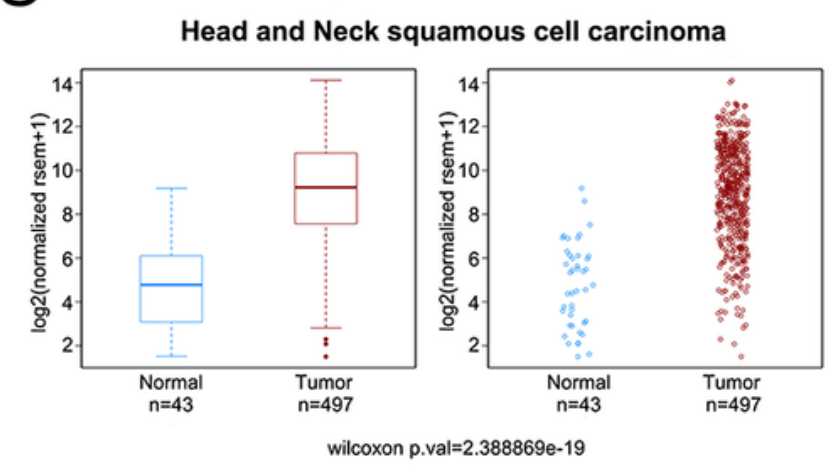

Expression of INHBA in

Breast invasive carcinoma

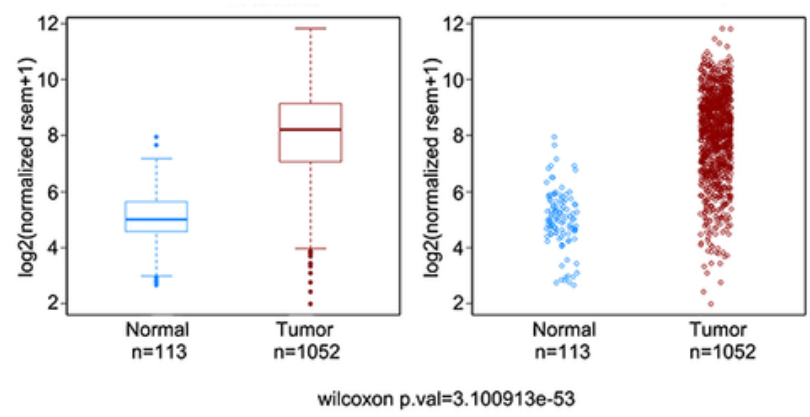

Figure 5 
INHBA is upregulated in ESCC, HNSC and invasive breast cancer. A. INHBA mRNA levels in ESCC and normal tissues analyzed using TCGA datasets. $\star \star \star \star P<0.0001$. B. Representative RISH staining of INHBA mRNA in ESCC and adjacent normal tissues. Scale bar $=200 \mu \mathrm{m}(100 x)$, scale bar $=100 \mu \mathrm{m}(200 \times)$. C. INHBA mRNA levels in HNSC, invasive breast cancer and normal tissues from TCGA database. ${ }^{\star \star \star *} \mathrm{P}<$ 0.0001 .

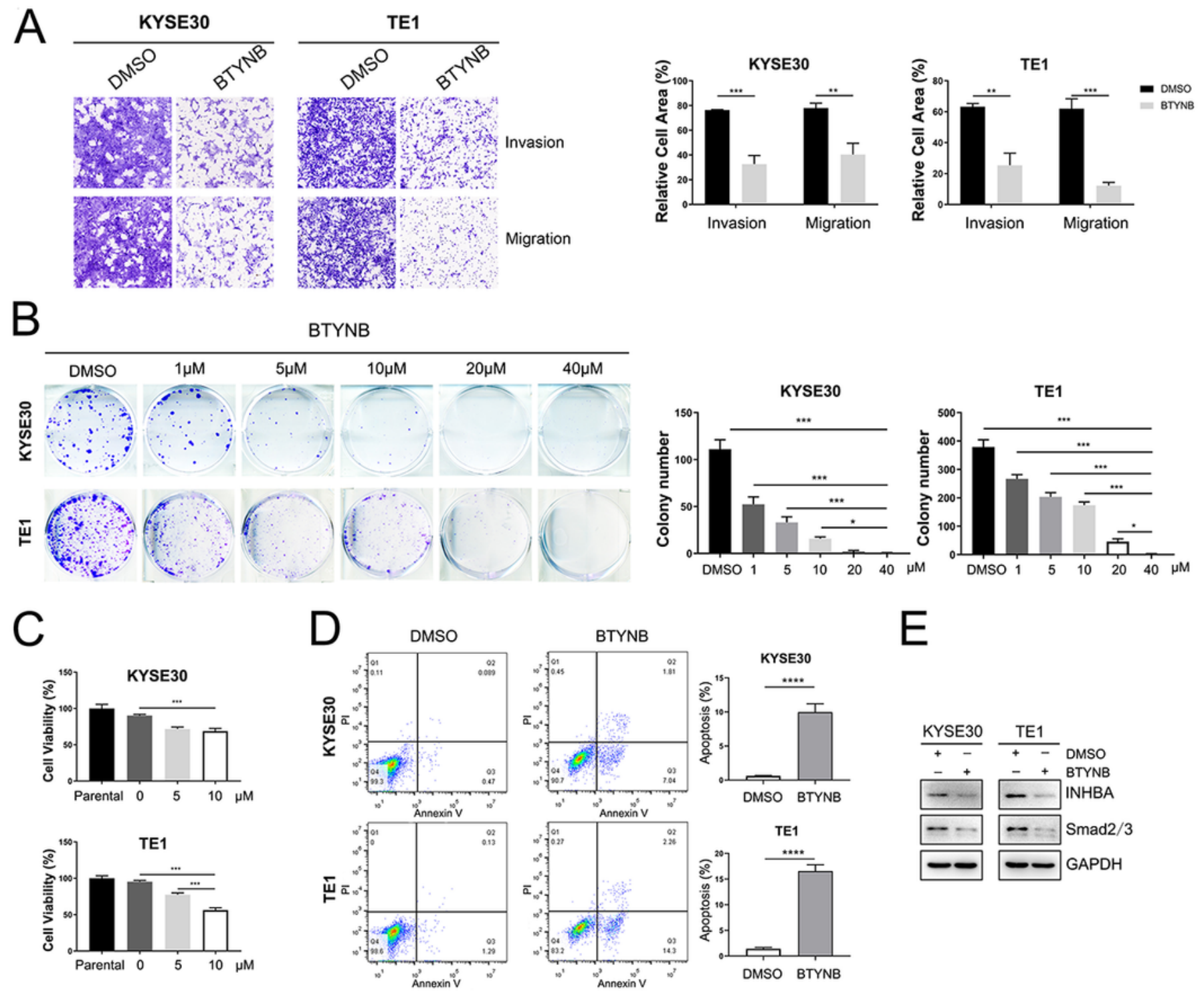

Figure 6 
BTYNB inhibits the invasion, migration and proliferation of ESCC cells in vitro. A. The effects of BTYNB on cell migration and invasion were examined by Transwell assay. BTYNB $(20 \mu \mathrm{M})$ was added to the lower compartment for $24 \mathrm{~h}-36 \mathrm{~h}$. Representative images (left) and statistical plots are shown (right). B. Colony formation of KYSE30 and TE1 cells exposed to different concentrations of BTYNB. Representative images of the indicated groups are shown in the left panel, and statistical plots are shown on the right. C. Cell viability of ESCC cells exposed to BTYNB was determined by CCK-8 assay. KYSE30 and TE1 cells were treated with $5 \mu \mathrm{M}$ and $10 \mu \mathrm{M}$ BTYNB for $72 \mathrm{~h}$. D. Cell apoptosis was determined by flow cytometry. Cells were treated with $10 \mu \mathrm{M}$ BTYNB for $48 \mathrm{~h}$. Representative images (left) and statistical plots are

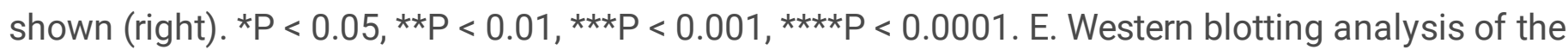
indicated proteins in cells treated with BTYNB. GAPDH was used as a loading control. 


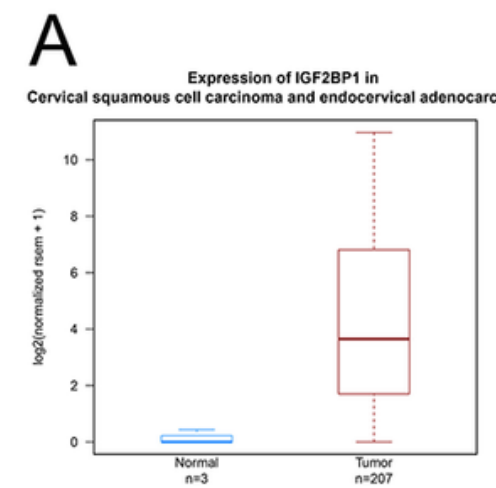

wilcoxon p.val = 5.2458730-03

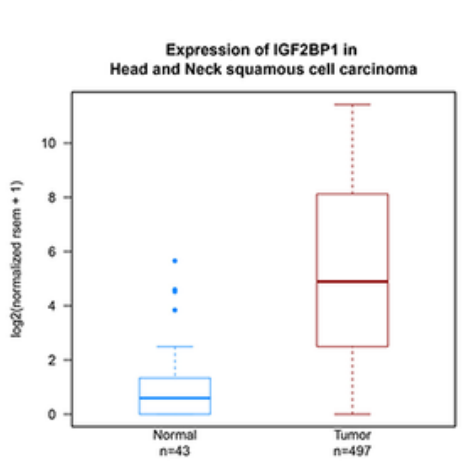

wileoxon p.val = 9.141578e-19

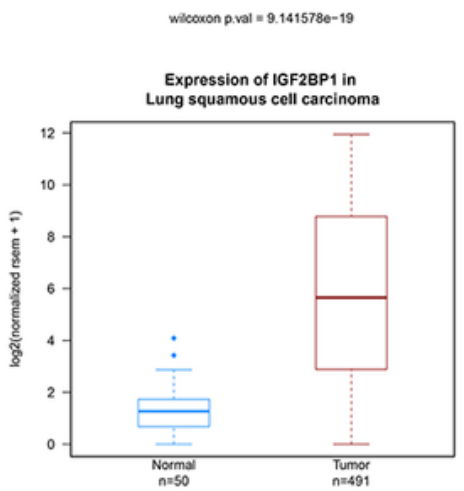

wilooxon pval $=4.0556030-20$

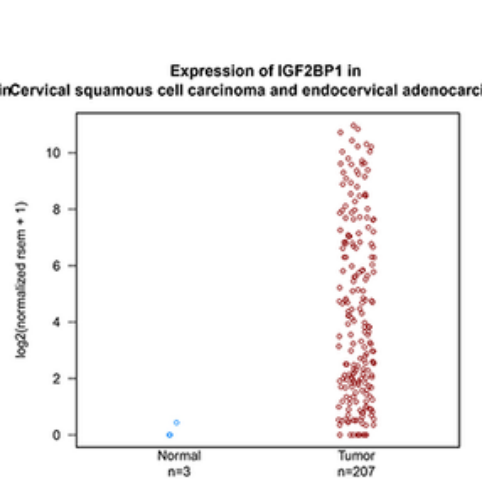

wicoxon p.val = 5.8459730-03

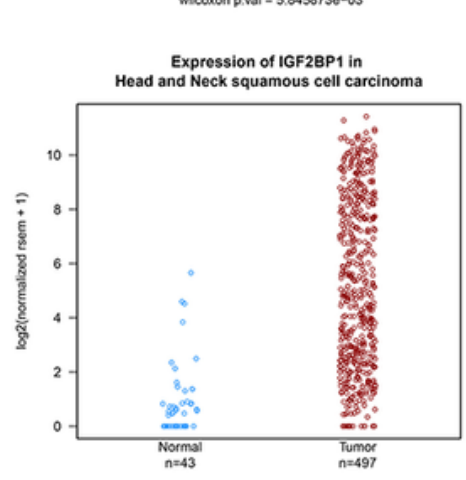

wilcoxon pval = 9.1415780-19

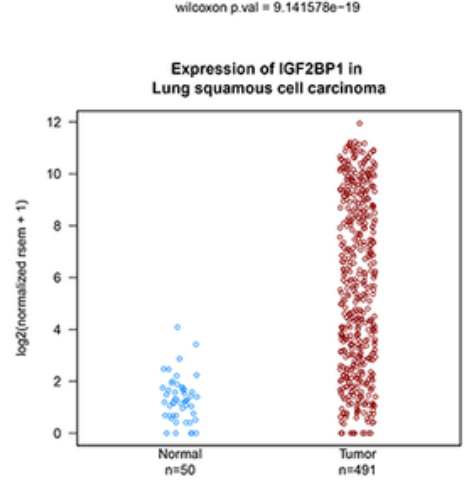

wilcoxon p.val $=4.0556030-20$
B

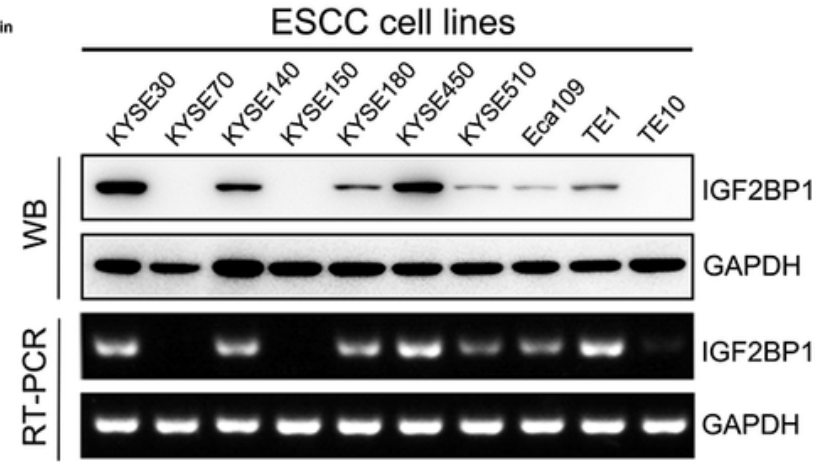

C

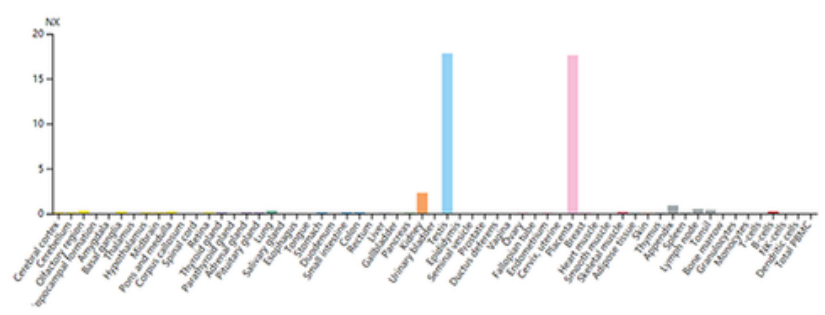

D

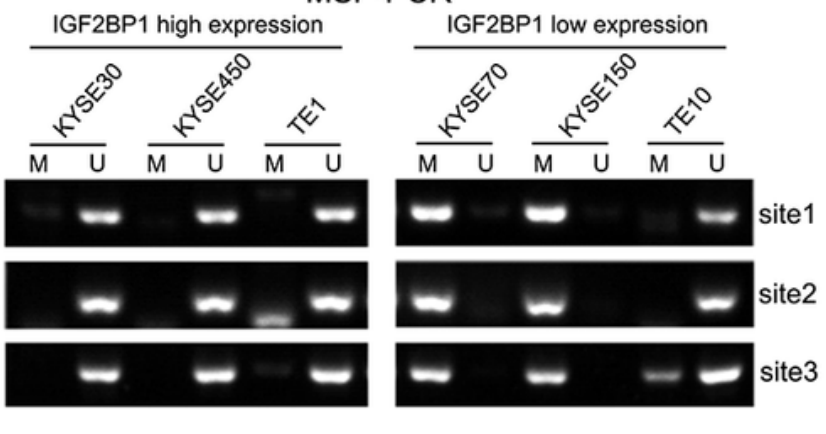

\section{Figure 7}

High IGF2BP1 expression in ESCC cells is associated with genomic hypomethylation. A. IGF2BP1 mRNA levels in HNSCC, LUSC, CESC patients and normal tissues in TCGA database. B. IGF2BP1 protein and mRNA levels of ESCC cell lines were analyzed using RT-PCR and Western blotting, respectively. C. The expression of IGF2BP1 mRNA in all major tissues and organs in the human body was analyzed in the HPA database. D. The methylation level of the first intron of the IGF2BP1 gene in the ESCC cell genome 
was assessed by MSP-PCR. Genomic DNA was extracted from ESCC cell lines with high and low IGF2BP1 expression. Three pairs of methylated and unmethylated primers ( $\mathrm{M}$ or $\mathrm{U}$ ) targeting three $\mathrm{CG}$ sites in the first intron were designed to amplify DNA converted by bisulfite.

\section{Supplementary Files}

This is a list of supplementary files associated with this preprint. Click to download.

- Additionalfile1SupplementaryMaterialsandMethods.docx

- Additionalfile2Figures1.eps

- Additionalfile3TableS7.xlsx

- Additionalfile4TableS8.xlsx

- Additionalfile5TableS9.xlsx

- Additionalfile6Figures2.eps

- Additionalfile7TableS10.xlsx

- Additionalfile8TableS11.xlsx

- Additionalfile9TableS12.xIsx 